\title{
The Radiation Problem from a Vertical Hertzian Dipole Antenna above Flat and Lossy Ground: Novel Formulation in the Spectral Domain with Closed-Form Analytical Solution in the High Frequency Regime
}

\author{
K. Ioannidi, ${ }^{1}$ Ch. Christakis, ${ }^{1}$ S. Sautbekov, ${ }^{2}$ P. Frangos, ${ }^{1}$ and S. K. Atanov ${ }^{2}$ \\ ${ }^{1}$ School of Electrical and Computer Engineering, National Technical University of Athens, 9 Iroon Polytechniou Street, \\ Zografou, 15773 Athens, Greece \\ ${ }^{2}$ Eurasian National University, 5 Munaitpasov Street, Astana 010008, Kazakhstan \\ Correspondence should be addressed to P. Frangos; pfrangos@central.ntua.gr
}

Received 6 March 2014; Revised 25 June 2014; Accepted 15 July 2014; Published 12 August 2014

Academic Editor: Dwight Jaggard

Copyright (C) $2014 \mathrm{~K}$. Ioannidi et al. This is an open access article distributed under the Creative Commons Attribution License, which permits unrestricted use, distribution, and reproduction in any medium, provided the original work is properly cited.

\begin{abstract}
We consider the problem of radiation from a vertical short (Hertzian) dipole above flat lossy ground, which represents the wellknown "Sommerfeld radiation problem" in the literature. The problem is formulated in a novel spectral domain approach, and by inverse three-dimensional Fourier transformation the expressions for the received electric and magnetic (EM) field in the physical space are derived as one-dimensional integrals over the radial component of wavevector, in cylindrical coordinates. This formulation appears to have inherent advantages over the classical formulation by Sommerfeld, performed in the spatial domain, since it avoids the use of the so-called Hertz potential and its subsequent differentiation for the calculation of the received EM field. Subsequent use of the stationary phase method in the high frequency regime yields closed-form analytical solutions for the received EM field vectors, which coincide with the corresponding reflected EM field originating from the image point. In this way, we conclude that the so-called "space wave" in the literature represents the total solution of the Sommerfeld problem in the high frequency regime, in which case the surface wave can be ignored. Finally, numerical results are presented, in comparison with corresponding numerical results based on Norton's solution of the problem.
\end{abstract}

\section{Introduction}

The so-called "Sommerfeld radiation problem" is a wellknown problem in the area of propagation of electromagnetic (EM) waves above flat lossy ground for obvious applications in the area of wireless telecommunications [1-6]. The classical Sommerfeld solution to this problem is provided in the physical space by using the so-called "Hertz potentials" and it does not end up with closed-form analytical solutions. Norton $[7,8]$ concentrated in subsequent years more on the engineering application of the above problem with obvious application to wireless telecommunications and provided approximate solutions to the above problem, which are represented by rather long algebraic expressions for engineering use, in which the so-called "attenuation coefficient" for the propagating surface wave plays an important role.

In this paper, the authors take advantage of previous research work of them for the EM radiation problem in free space [9] by using the spectral domain approach. Furthermore, in [10], the authors provided the fundamental formulation for the problem considered here, that is, the solution in spectral domain for the radiation from a dipole moment at a specific angular frequency $(\omega)$ in isotropic media with a flat infinite interface. In that paper, the authors end up with integral representations for the received electric and magnetic fields above or below the interface (line-of-sight (LOS) plus reflected field-transmitted fields, resp.), where the integration takes place over the radial spectral coordinate 
$k_{\rho}$. Then, in the present paper, the authors concentrate on the solution of the classical "Sommerfeld radiation problem" described above, where the radiation of a vertical dipole moment at angular frequency $\omega$ takes place above flat lossy ground (this is equivalent to the radiation of a vertical small (Hertzian) dipole above flat lossy ground, as it will be explained by formula in the main text). By using the stationary phase method (SPM method [11-13]), integration over the radial spectral coordinate $k_{\rho}$ is performed and the high frequency solution to the problem ("space wave," which represents the interference of the line-of-sight (LOS) and the wave scattered from the ground) is derived, as it will be explained in detail in Section 4. Finally, numerical results which show both the "space wave" mentioned above and Norton's "surface wave" $[7,8]$ are presented in Section 6. A shorter version of the present paper of ours can be found in [14].

\section{Geometry of the Radiation Problem}

The geometry of the problem is given in Figure 1. Here, a Hertzian (small) dipole with dipole moment $p$ directed to positive $x$-axis, at altitude $x_{0}$ above the infinite, flat, and lossy ground, radiates time-harmonic electromagnetic (EM) waves at angular frequency $\omega=2 \pi f(\exp (-i \omega t)$ time dependence is assumed in this paper). Here, the relative complex permittivity of the ground (medium 2) is $\varepsilon_{r}^{\prime}=$ $\varepsilon^{\prime} / \varepsilon_{0}=\varepsilon_{r}+i x$, where $x=\sigma / \omega \varepsilon_{0}=18 \times 10^{9} \sigma / f$, with $\sigma$ being the ground conductivity and $f$ the frequency of radiation, and $\varepsilon_{0}=8.854 \times 10^{-12} \mathrm{~F} / \mathrm{m}$ is the absolute permittivity in vacuum or air. Then, the wavenumbers of propagation of EM waves in air and lossy ground, respectively, are given by the following:

$$
\begin{aligned}
& k_{01}=\frac{\omega}{c_{1}}=\omega \sqrt{\varepsilon_{1} \mu_{1}}=\omega \sqrt{\varepsilon_{0} \mu_{0} \varepsilon_{r 1} \mu_{r 1}}=\omega \sqrt{\varepsilon_{0} \mu_{0}}, \\
& k_{02}=\frac{\omega}{c_{2}}=\omega \sqrt{\varepsilon_{2} \mu_{2}}=\omega \sqrt{\varepsilon_{r 2} \mu_{r 2} \varepsilon_{0} \mu_{0}}=k_{01} \sqrt{\varepsilon_{r}+i x} .
\end{aligned}
$$

The Maxwell equations for the time-harmonic EM fields considered above are given by

$$
\begin{aligned}
& \operatorname{rot} \underline{E}-i \omega \mu_{0} \mu_{r} \underline{H}=0, \\
& \operatorname{rot} \underline{H}+i \omega \varepsilon_{0} \varepsilon_{r} \underline{H}=\underline{j},
\end{aligned}
$$

where $j$ is current density (source of EM fields considered here).

\section{Formulation of the Sommerfeld Radiation Problem in the Spectral Domain: Integral Representation for the Received Electric and Magnetic Fields}

3.1. EM Fields in terms of Spectral Domain Current Densities. Following $[9,10]$, the EM field in physical space is derived from current density $\widetilde{J}$ in spectral domain and Green's

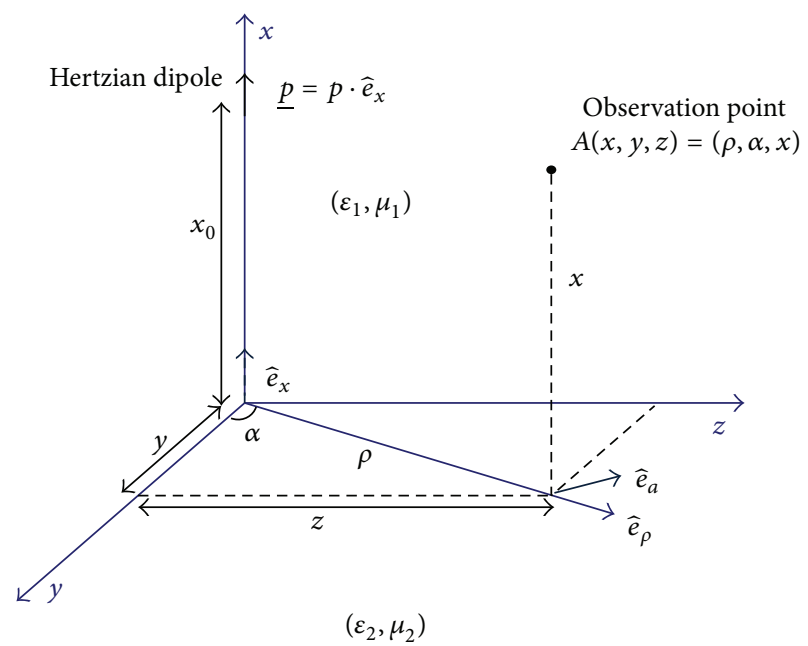

FIGURE 1: Geometry of the radiation problem considered in this paper. The radiating dipole is at position $\left(x_{0}, 0,0\right)$ above infinite, flat, and lossy ground situated at plane $x=0$.

function $\widetilde{\psi}$, also in the spectral domain, through inverse three-dimensional (3D) Fourier transformation as follows:

$$
\begin{gathered}
\underline{H}=-i F^{-1}[\widetilde{\psi} \cdot(\underline{k} \times \widetilde{J})], \\
\underline{E}=-\frac{i}{\omega \varepsilon_{r} \varepsilon_{0}} F^{-1}\left\{\widetilde{\psi}\left[\varepsilon_{r} \mu_{r} k_{0}^{2} \widetilde{I}-\langle\underline{k}, \underline{J}\rangle \underline{k}\right]\right\},
\end{gathered}
$$

where the symbol \langle\rangle denotes the inner product, $F^{-1}$ is the inverse 3D Fourier transform (FT) operator, and

$$
\widetilde{\psi}=\left(k_{01}^{2}-k^{2}\right)^{-1}=\left(k_{01}^{2}-k_{\rho}^{2}-k_{x}^{2}\right)^{-1}
$$

is 3D Green's function in spectral domain and cylindrical coordinates. Furthermore, by noting that, for the problem considered here, current density $\widetilde{J}=\left[\widetilde{J}\left(k_{\rho}\right), 0,0\right]$ has only $x$ component and that wavevector $\underline{\underline{k}}=\left(k_{\rho}, k_{\alpha}=0, k_{x}\right)$ does not possess azimuthal $\alpha$ component, by performing the cross product and inverse FT operation of (3), we obtain

$$
\begin{array}{r}
\underline{H}(\underline{r})=-\frac{i}{(2 \pi)^{3}} \widehat{e}_{\alpha} \int_{k_{\rho}=0}^{\infty} \int_{\alpha=0}^{2 \pi} \int_{k_{x}=-\infty}^{\infty} k_{x} \widetilde{J}\left(k_{\rho}\right) \widetilde{\psi} k_{\rho} \\
\cdot \exp (i \underline{k} \cdot \underline{r}) d k_{\rho} d \alpha d k_{x} .
\end{array}
$$

Similarly, by performing the inner product and inverse FT operation of (4), we obtain

$$
\begin{aligned}
& \underline{E}(\underline{r})=-\frac{i}{(2 \pi)^{3} \varepsilon_{r} \varepsilon_{0} \omega} \int_{0}^{\infty} \int_{0}^{2 \pi} \int_{-\infty}^{\infty}\left(\varepsilon_{r} \mu_{r} k_{0}^{2} \widehat{e}_{\rho}-k_{\rho} \underline{k}\right) \\
& \cdot \widetilde{J}\left(k_{\rho}\right) \widetilde{\psi} k_{\rho} \\
& \times \exp (i \underline{k} \cdot \underline{r}) d k_{\rho} d \alpha d k_{x},
\end{aligned}
$$


where

$$
\underline{k}=\left(k_{\rho}, 0, k_{x}\right)=k_{\rho} \widehat{e}_{\rho}+k_{x} \widehat{e}_{x}
$$

is the wavevector of propagation and $\underline{r}=(\rho, \alpha, x)$ is the point of observation (see Figure 1), all in cylindrical coordinates. Furthermore, by taking (8) into account, (7) for the received electric field can also be written in the following form:

$$
\begin{array}{r}
\underline{E}(\underline{r})=-\frac{i}{(2 \pi)^{3} \varepsilon_{r} \varepsilon_{0} \omega} \int_{0}^{\infty} \int_{0}^{2 \pi} \int_{-\infty}^{\infty}( \\
\left.\left(\varepsilon_{r} \mu_{r} k_{0}^{2}-k_{\rho}^{2}\right) \widehat{e}_{\rho}-k_{\rho} k_{x} \widehat{e}_{x}\right) \\
\cdot \widetilde{J}\left(k_{\rho}\right) \widetilde{\psi} k_{\rho} \\
\times \exp (i \underline{k} \cdot \underline{r}) d k_{\rho} d \alpha d k_{x} .
\end{array}
$$

Furthermore, in order to integrate expressions (6) and (9) with respect to azimuthal angle $\alpha$ (see Figure 1), we take into account the fact that

$$
\underline{k} \cdot \underline{r}=k_{x} x+k_{\rho} \rho \cdot \cos (\alpha-\beta),
$$

where $\beta$ is the azimuth angle of the projection of vector $\underline{k}$ on the $y z$-plane (see Figure 1). Then, by using the following identities for Bessel functions:

$$
\begin{gathered}
\frac{1}{2 \pi} \int_{0}^{2 \pi} \exp \left(i k_{\rho} \rho \cos \alpha\right) d \alpha=J_{0}\left(k_{\rho} \rho\right), \\
\int_{0}^{\infty} J_{0}\left(k_{\rho} \rho\right) d k_{\rho}=\frac{1}{2} \int_{-\infty}^{\infty} H_{0}^{(1)}\left(k_{\rho} \rho\right) d k_{\rho}
\end{gathered}
$$

where $J_{0}$ is the Bessel function of first kind and zero order and $H_{0}^{(1)}$ is the Hankel function of first kind and zero order, we obtain

$$
\begin{gathered}
\underline{H}(\underline{r})=-\frac{i}{8 \pi^{2}} \widehat{e}_{\alpha} \int_{k_{\rho}=-\infty}^{\infty} \int_{k_{x}=-\infty}^{\infty} k_{x} \widetilde{J}\left(k_{\rho}\right) \tilde{\psi} k_{\rho} \\
\cdot H_{0}^{(1)}\left(k_{\rho} \rho\right) \exp \left(i k_{x} x\right) d k_{\rho} d k_{x}, \\
\underline{E}(\underline{r})=-\frac{i}{8 \pi^{2} \omega \varepsilon_{r} \varepsilon_{0}} \int_{k_{\rho}=-\infty}^{\infty} \int_{k_{x}=-\infty}^{\infty}\left(\left(\varepsilon_{r} \mu_{r} k_{0}^{2}-k_{\rho}^{2}\right) \hat{e}_{\rho}\right. \\
\left.-k_{\rho} k_{x} \widehat{e}_{x}\right) \\
\times \widetilde{J}\left(k_{\rho}\right) \widetilde{\psi} k_{\rho} H_{0}^{(1)}\left(k_{\rho} \rho\right) \\
\times \exp \left(i k_{x} x\right) d k_{\rho} d k_{x} .
\end{gathered}
$$

3.2. Formulation of the Boundary Value Problem. For the problem considered in this work (Figure 1), we now use (12), to write the appropriate expressions for the reflected $(R)$ and transmitted (T) EM field, as follows:

$$
\begin{aligned}
& \underline{H}^{R}(\underline{r})=-\frac{i}{8 \pi^{2}} \widehat{e}_{\alpha} \int_{k_{\rho}=-\infty}^{\infty} \int_{k_{x}=-\infty}^{\infty} k_{x} \widetilde{J}_{R}\left(k_{\rho}\right) \tilde{\psi}_{1} k_{\rho} \\
& \cdot H_{0}^{(1)}\left(k_{\rho} \rho\right) \\
& \times \exp \left(i k_{x} x\right) d k_{\rho} d k_{x} \\
& \underline{E}^{R}(\underline{r})=-\frac{i}{8 \pi^{2} \omega \varepsilon_{r 1} \varepsilon_{0}} \int_{k_{\rho}=-\infty}^{\infty} \int_{k_{x}=-\infty}^{\infty}\left(\left(\varepsilon_{r 1} \mu_{r 1} k_{01}^{2}-k_{\rho}^{2}\right) \hat{e}_{\rho}\right. \\
& \left.-k_{\rho} k_{x} \widehat{e}_{x}\right) \\
& \cdot \widetilde{J}_{R}\left(k_{\rho}\right) \widetilde{\psi}_{1} k_{\rho} H_{0}^{(1)}\left(k_{\rho} \rho\right) \\
& \times \exp \left(i k_{x} x\right) d k_{\rho} d k_{x} \\
& \underline{H}^{T}(\underline{r})=-\frac{i}{8 \pi^{2}} \widehat{e}_{\alpha} \int_{k_{\rho}=-\infty}^{\infty} \int_{k_{x}=-\infty}^{\infty} k_{x} \widetilde{J}_{T}\left(k_{\rho}\right) \widetilde{\psi}_{2} k_{\rho} \\
& \cdot H_{0}^{(1)}\left(k_{\rho} \rho\right) \\
& \times \exp \left(i k_{x} x\right) d k_{\rho} d k_{x} \\
& \underline{E}^{T}(\underline{r})=-\frac{i}{8 \pi^{2} \omega \varepsilon_{r 2} \varepsilon_{0}} \int_{k_{\rho}=-\infty}^{\infty} \int_{k_{x}=-\infty}^{\infty}\left(\left(\varepsilon_{r 2} \mu_{r 2} k_{02}^{2}-k_{\rho}^{2}\right) \widehat{e}_{\rho}\right. \\
& \left.-k_{\rho} k_{x} \widehat{e}_{x}\right) \\
& \text { - } \widetilde{J}_{T}\left(k_{\rho}\right) \widetilde{\psi}_{2} k_{\rho} H_{0}^{(1)}\left(k_{\rho} \rho\right) \\
& \times \exp \left(i k_{x} x\right) d k_{\rho} d k_{x} \text {, }
\end{aligned}
$$

where $k_{01}$ and $k_{02}$ are given by (1) and

$$
\begin{aligned}
& \widetilde{\psi}_{1}=\frac{1}{k_{01}^{2}-k_{\rho}^{2}-k_{x}^{2}}, \\
& \widetilde{\psi}_{2}=\frac{1}{k_{02}^{2}-k_{\rho}^{2}-k_{x}^{2}} .
\end{aligned}
$$

$\underline{\tilde{J}_{R}}=\left[\widetilde{J}_{R}\left(k_{\rho}\right), 0,0\right], \underline{\tilde{J}_{T}}=\left[\widetilde{J}_{T}\left(k_{\rho}\right), 0,0\right]$ are the Fourier components of surface current density. Furthermore, the line-ofsight (LOS) EM field of the Hertzian dipole in thefar field is given by $[11,15]$

$$
\begin{aligned}
H_{\alpha}^{\mathrm{LOS}}(r, \theta) & =\frac{\omega^{2} p}{4 \pi} \sqrt{\varepsilon_{0} \mu_{0}} \frac{\exp (i k r)}{r} \sin \theta \\
& =\frac{\omega k_{01} p}{4 \pi} \frac{\exp (i k r)}{r} \sin \theta,
\end{aligned}
$$

where spherical coordinates $(r, \theta)$ are given in terms of cylindrical coordinates $(\rho, x)$ (see Figure 1 ) by

$$
r \approx \rho+\frac{\left(x-x_{0}\right)^{2}}{2 \rho},
$$




$$
\theta=\pi-\tan ^{-1}\left[\frac{\rho}{\left(x_{0}-x\right)}\right], \quad \text { for } x_{0}>x
$$

or

$$
\begin{gathered}
\theta=\tan ^{-1}\left[\frac{\rho}{\left(x-x_{0}\right)}\right], \quad \text { for } x>x_{0}, \\
\underline{E}^{\mathrm{LOS}}(r, \theta)=\zeta H_{\alpha}^{\mathrm{LOS}} \cos \theta \widehat{e}_{\rho}-\zeta H_{\alpha}^{\mathrm{LOS}} \sin \theta \widehat{e}_{x},
\end{gathered}
$$

where $H_{\alpha}^{\mathrm{LOS}}$ is given by (15)-((17a) and (17b)).

Then, the total EM field in the regions $x>0$ and $x<0$ (see Figure 1) is given by

$$
\begin{aligned}
& \underline{H}(\underline{r})= \begin{cases}\underline{H}^{\mathrm{LOS}}(\underline{r})+\underline{H}^{R}(\underline{r}), & x>0, \\
\underline{H}^{T}(\underline{r}), & x<0,\end{cases} \\
& \underline{E}(\underline{r})= \begin{cases}\underline{E}^{\mathrm{LOS}}(\underline{r})+\underline{E}^{R}(\underline{r}), & x>0, \\
\underline{E}^{T}(\underline{r}), & x<0 .\end{cases}
\end{aligned}
$$

Furthermore, by performing the integrations of expressions (13) over $k_{x}$, by using the residue theorem [16], we obtain the following integral expressions for the EM fields.

In the upper half space $(x>0)$,

$$
\begin{aligned}
& \underline{H}(\underline{r})= \underline{H}^{\mathrm{LOS}}(\underline{r})-\frac{\widehat{e}_{\alpha}}{8 \pi} \int_{-\infty}^{\infty} k_{\rho} \widetilde{J}_{R}\left(k_{\rho}\right) H_{0}^{(1)}\left(k_{\rho} \rho\right) e^{i \kappa_{1} x} d k_{\rho}, \\
& \underline{E}(\underline{r})= \underline{E}^{\mathrm{LOS}}(\underline{r})-\frac{1}{8 \pi \omega \varepsilon_{r 1} \varepsilon_{0}} \widehat{e}_{\rho} \int_{-\infty}^{\infty} \kappa_{1} k_{\rho} \widetilde{J}_{R}\left(k_{\rho}\right) \\
& \cdot H_{0}^{(1)}\left(k_{\rho} \rho\right) e^{i k_{1} x} d k_{\rho} \\
&+\frac{1}{8 \pi \omega \varepsilon_{r 1} \varepsilon_{0}} e_{x} \int_{-\infty}^{\infty} k_{\rho}^{2} \widetilde{J}_{R}\left(k_{\rho}\right) H_{0}^{(1)}\left(k_{\rho} \rho\right) e^{i \kappa_{1} x} d k_{\rho},
\end{aligned}
$$

while for the lower half space $(x<0)$

$$
\begin{gathered}
\underline{H}^{T}(\underline{r})=\frac{\widehat{e}_{\alpha}}{8 \pi} \int_{-\infty}^{\infty} k_{\rho} \widetilde{J}_{T}\left(k_{\rho}\right) H_{0}^{(1)}\left(k_{\rho} \rho\right) e^{-i \kappa_{2} x} d k_{\rho} \\
\underline{E}^{T}(\underline{r})=-\frac{1}{8 \pi \omega \varepsilon_{r 2} \varepsilon_{0}} \widehat{e}_{\rho} \int_{-\infty}^{\infty} \kappa_{2} k_{\rho} \widetilde{J}_{T}\left(k_{\rho}\right) \cdot H_{0}^{(1)}\left(k_{\rho} \rho\right) e^{-i k_{2} x} d k_{\rho} \\
+\frac{1}{8 \pi \omega \varepsilon_{r 2} \varepsilon_{0}} \widehat{e}_{x} \int_{-\infty}^{\infty} k_{\rho}^{2} \widetilde{J}_{T}\left(k_{\rho}\right) H_{0}^{(1)}\left(k_{\rho} \rho\right) e^{-i k_{2} x} d k_{\rho}
\end{gathered}
$$

where

$$
\begin{aligned}
& \kappa_{1}=\sqrt{k_{01}^{2}-k_{\rho}^{2}}, \\
& \kappa_{2}=\sqrt{k_{02}^{2}-k_{\rho}^{2}} .
\end{aligned}
$$

3.3. Application of the Boundary Conditions (BCs): Solution for the Unknown Current Densities at the Interface in Spectral Domain. We now apply the BCs that at the interface $(x=0)$ the tangential components of electric field $E$ and magnetic field $H$ must be continuous; namely,

$$
\begin{gathered}
H_{\alpha}^{\mathrm{LOS}}+H_{\alpha}^{R}=H_{\alpha}^{T}, \\
E_{\rho}^{\mathrm{LOS}}+E_{\rho}^{R}=E_{\rho}^{T},
\end{gathered}
$$

where

$$
\begin{aligned}
H_{\alpha}^{\mathrm{LOS}} & =-\frac{1}{8 \pi} \int_{-\infty}^{\infty} \frac{i \omega p k_{\rho}^{2} e^{i \kappa_{1} x_{0}}}{\kappa_{1}} H_{0}^{(1)}\left(k_{\rho} \rho\right) d k_{\rho}, \\
E_{\rho}^{\mathrm{LOS}} & =\frac{1}{8 \pi \varepsilon_{r 1} \varepsilon_{0}} \int_{-\infty}^{\infty} i \omega p k_{\rho}^{2} e^{i \kappa_{1} x_{0}} H_{0}^{(1)}\left(k_{\rho} \rho\right) d k_{\rho}, \\
H^{R} & =-\frac{1}{8 \pi} \int_{-\infty}^{\infty} k_{\rho} \widetilde{J}_{R}\left(k_{\rho}\right) H_{0}^{(1)}\left(k_{\rho} \rho\right) d k_{\rho}, \\
E_{\rho}^{R} & =-\frac{1}{8 \pi \omega \varepsilon_{r 1} \varepsilon_{0}} \int_{-\infty}^{\infty} \kappa_{1} k_{\rho} \widetilde{J}_{R}\left(k_{\rho}\right) H_{0}^{(1)}\left(k_{\rho} \rho\right) d k_{\rho}, \\
H_{\alpha}^{T} & =\frac{1}{8 \pi} \int_{-\infty}^{\infty} k_{\rho} \widetilde{J}_{T}\left(k_{\rho}\right) H_{0}^{(1)}\left(k_{\rho} \rho\right) d k_{\rho}, \\
\underline{E}^{T}(\underline{r}) & =-\frac{1}{8 \pi \omega \varepsilon_{r 2} \varepsilon_{0}} \int_{-\infty}^{\infty} \kappa_{2} k_{\rho} \widetilde{J}_{T}\left(k_{\rho}\right) H_{0}^{(1)}\left(k_{\rho} \rho\right) d k_{\rho} .
\end{aligned}
$$

Then, from (23), we find

$$
\begin{aligned}
& \frac{1}{8 \pi} \int_{-\infty}^{\infty}\left(\frac{i \omega p k_{\rho} e^{i \kappa_{1} x_{0}}}{\kappa_{1}}+\widetilde{J}_{R}\left(k_{\rho}\right)\right) H_{0}^{(1)}\left(k_{\rho} \rho\right) k_{\rho} d k_{\rho} \\
& \quad=-\frac{1}{8 \pi} \int_{-\infty}^{\infty} \tilde{J}_{T}\left(k_{\rho}\right) H_{0}^{(1)}\left(k_{\rho} \rho\right) k_{\rho} d k_{\rho}, \\
& \frac{1}{8 \pi \varepsilon_{r 1} \varepsilon_{0}} \int_{-\infty}^{\infty}\left(-i \omega p k_{\rho} e^{i \kappa_{1} x_{0}}+\widetilde{J}_{R}\left(k_{\rho}\right) \kappa_{1}\right) \cdot H_{0}^{(1)}\left(k_{\rho} \rho\right) k_{\rho} d k_{\rho} \\
& =\frac{1}{8 \pi \varepsilon_{r 2} \varepsilon_{0}} \int_{-\infty}^{\infty} \tilde{J}_{T}\left(k_{\rho}\right) \kappa_{2} H_{0}^{(1)}\left(k_{\rho} \rho\right) k_{\rho} d k_{\rho} .
\end{aligned}
$$

Therefore, from (25), we obtain the following system of algebraic equations:

$$
\begin{gathered}
\frac{i \omega p k_{\rho} e^{i \kappa_{1} x_{0}}}{\kappa_{1}}+\widetilde{J}_{R}\left(k_{\rho}\right)=-\widetilde{J}_{T}\left(k_{\rho}\right), \\
-i \omega p k_{\rho} e^{i \kappa_{1} x_{0}}+\widetilde{J}_{R}\left(k_{\rho}\right) \kappa_{1}=\frac{\varepsilon_{r 1}}{\varepsilon_{r 2}} \widetilde{J}_{T}\left(k_{\rho}\right) \kappa_{2} .
\end{gathered}
$$

The solutions of systems of (26a) and (26b) are the unknown Fourier components of surface current densities, as follows:

$$
\begin{gathered}
\widetilde{J}_{R}\left(k_{\rho}\right)=i \omega p k_{\rho} e^{i \kappa_{1} x_{0}} \frac{\varepsilon_{r 2} \kappa_{1}-\varepsilon_{r 1} \kappa_{2}}{\kappa_{1}\left(\varepsilon_{r 2} \kappa_{1}+\varepsilon_{r 1} \kappa_{2}\right)}, \\
\tilde{J}_{T}\left(k_{\rho}\right)=-i \omega p k_{\rho} e^{i \kappa_{1} x_{0}} \frac{2 \varepsilon_{r 2}}{\varepsilon_{r 2} \kappa_{1}+\varepsilon_{r 1} \kappa_{2}} .
\end{gathered}
$$


3.4. Expressions for the Reflected and Transmitted EM Fields in Integral Representations. Substituting expressions of (27a) and (27b) for the unknown current densities (at the interface, in spectral domain) in (20)-(21), we obtain the reflected and transmitted EM fields in integral representations, as follows.

In the higher half space (LOS field plus reflected field, $x>0$ ),

$$
\begin{gathered}
\underline{H}(\underline{r})=\underline{H}^{\mathrm{LOS}}-\frac{i \omega p \widehat{e}_{\alpha}}{8 \pi} \int_{-\infty}^{\infty} \frac{\varepsilon_{r 2} \kappa_{1}-\varepsilon_{r 1} \kappa_{2}}{\kappa_{1}\left(\varepsilon_{r 2} \kappa_{1}+\varepsilon_{r 1} \kappa_{2}\right)} k_{\rho}^{2} \\
\cdot H_{0}^{(1)}\left(k_{\rho} \rho\right) e^{i \kappa_{1}\left(x_{0}+x\right)} d k_{\rho}, \\
\underline{E}(\underline{r})=\underline{E}^{\mathrm{LOS}}(\underline{r})-\frac{i p}{8 \pi \varepsilon_{r 1} \varepsilon_{0}} \widehat{e}_{\rho} \int_{-\infty}^{\infty} k_{\rho}^{2} \frac{\varepsilon_{r 2} \kappa_{1}-\varepsilon_{r 1} \kappa_{2}}{\left(\varepsilon_{r 2} \kappa_{1}+\varepsilon_{r 1} \kappa_{2}\right)} \\
+\frac{i p}{8 \pi \varepsilon_{r 1} \varepsilon_{0}} \widehat{e}_{x} \int_{-\infty}^{\infty \kappa_{1}\left(x+x_{0}\right)} H_{0}^{(1)}\left(k_{\rho} \rho\right) d k_{\rho}^{3} \frac{\varepsilon_{r 2} \kappa_{1}-\varepsilon_{r 1} \kappa_{2}}{\kappa_{1}\left(\varepsilon_{r 2} \kappa_{1}+\varepsilon_{r 1} \kappa_{2}\right)} e^{i \kappa_{1}\left(x+x_{0}\right)} \\
\cdot H_{0}^{(1)}\left(k_{\rho} \rho\right) d k_{\rho} .
\end{gathered}
$$

The physical interpretation of (28), which represent one of the main results of this paper, is that the scattered EM field at the observation point consists of a complex summation of the EM waves scattered from the different points of the flat and lossy ground, each one with its own local reflection coefficient (here, the term "complex summation" means that the amplitude and phase of these individual scattered waves must be taken into account).

In the lower half space (transmitted fields, $x<0$ ),

$$
\begin{gathered}
\underline{H}^{T}(\underline{r})=-\frac{i \omega p}{4 \pi} \widehat{e}_{\alpha} \int_{-\infty}^{\infty} k_{\rho}^{2} \frac{\varepsilon_{r 2}}{\varepsilon_{r 2} \kappa_{1}+\varepsilon_{r 1} \kappa_{2}} e^{i\left(\kappa_{1} x_{0}-\kappa_{2} x\right)} \\
\cdot H_{0}^{(1)}\left(k_{\rho} \rho\right) d k_{\rho}, \\
\underline{E}^{T}(\underline{r})=-\frac{i p}{4 \pi \varepsilon_{0}} \int_{-\infty}^{\infty}\left(k_{\rho} \widehat{e}_{x}-\kappa_{2} \widehat{e}_{\rho}\right) \frac{k_{\rho}^{2}}{\varepsilon_{r 2} \kappa_{1}+\varepsilon_{r 1} \kappa_{2}} \\
\cdot e^{i\left(\kappa_{1} x_{0}-\kappa_{2} x\right)} H_{0}^{(1)}\left(k_{\rho} \rho\right) d k_{\rho} .
\end{gathered}
$$

\section{Electromagnetic (EM) Fields Reflected from Infinite, Flat, and Lossy Ground in the Far Field Region: Analytical High Frequency Expressions Obtained through the Application of the Stationary Phase Method (SPM)}

In order to calculate the EM field above lossy ground (i.e., for $x>0$ ), we write (28) in the following form:

$$
\begin{aligned}
& \underline{E}_{x>0}=\underline{E}^{\mathrm{LOS}}-\frac{i p}{8 \pi \varepsilon_{0} \varepsilon_{r 1}} I_{1} \cdot \hat{e}_{\rho}-\frac{i p}{8 \pi \varepsilon_{0} \varepsilon_{r 1}} I_{2} \cdot \widehat{e}_{x}, \\
& \underline{H}_{x>0}=\underline{H}^{\mathrm{LOS}}-\frac{i \omega p}{8 \pi} I_{3} \cdot \hat{e}_{\alpha},
\end{aligned}
$$

where

$$
I_{1}=\int_{k_{\rho}=-\infty}^{\infty} \frac{\varepsilon_{2} \kappa_{1}-\varepsilon_{1} \kappa_{2}}{\varepsilon_{2} \kappa_{1}+\varepsilon_{1} \kappa_{2}} \cdot k_{\rho}^{2} \cdot H_{0}^{(1)}\left(k_{\rho} \rho\right) \cdot e^{i \kappa_{1}\left(x+x_{0}\right)} d k_{\rho},
$$

$$
\begin{aligned}
& I_{2}=\int_{k_{\rho}=-\infty}^{\infty} \frac{k_{\rho}\left(\varepsilon_{2} \kappa_{1}-\varepsilon_{1} \kappa_{2}\right)}{\kappa_{1}\left(\varepsilon_{2} \kappa_{1}+\varepsilon_{1} \kappa_{2}\right)} \cdot k_{\rho}^{2} \cdot H_{0}^{(1)}\left(k_{\rho} \rho\right) \cdot e^{i \kappa_{1}\left(x+x_{0}\right)} d k_{\rho}, \\
& I_{3}=\int_{k_{\rho}=-\infty}^{\infty} \frac{\varepsilon_{2} \kappa_{1}-\varepsilon_{1} \kappa_{2}}{\kappa_{1}\left(\varepsilon_{2} \kappa_{1}+\varepsilon_{1} \kappa_{2}\right)} \cdot k_{\rho}^{2} \cdot H_{0}^{(1)}\left(k_{\rho} \rho\right) \cdot e^{i \kappa_{1}\left(x+x_{0}\right)} d k_{\rho} .
\end{aligned}
$$

Furthermore, in order to calculate integral $I_{1}$ (in an almost identical manner, integrals $I_{2}$ and $I_{3}$ will be calculated, using the SPM method [11-13, 17]), let us assume large argument approximation for the Hankel functions of (32)(34); namely, let us assume that

$$
k_{\rho} \cdot \rho \gg 1
$$

for which case function $H_{0}^{(1)}\left(k_{\rho} \rho\right)$ becomes a highly oscillating function of $k_{\rho}$. Then, since stationary phase method (SPM) is to be applied, we just replace $H_{0}^{(1)}\left(k_{\rho} \rho\right)$ in (32) by its asymptotic large argument approximation:

$$
H_{0}^{(1)}\left(k_{\rho} \rho\right)=\sqrt{\frac{-2 i}{\pi k_{\rho} \rho}} \cdot e^{+i k_{\rho} \rho} .
$$

Then, integral $I_{1}$ of (32) takes the following form:

$$
I_{1}=\sqrt{\frac{-2 i}{\pi}} \cdot \frac{1}{\sqrt{\rho}} \int_{k_{\rho}=-\infty}^{\infty} k_{\rho}^{3 / 2} \cdot \frac{\varepsilon_{2} \kappa_{1}-\varepsilon_{1} \kappa_{2}}{\varepsilon_{2} \kappa_{1}+\varepsilon_{1} \kappa_{2}} \cdot e^{i \kappa_{1}\left(x+x_{0}\right)} e^{+i k_{\rho} \rho} d k_{\rho}
$$

Moreover, in order to apply SPM method, we define radial distance $\rho$ (see Figure 1) as "large parameter," and we also define the following.

Phase function is

$$
f\left(k_{\rho}\right)=\frac{\kappa_{1}\left(x+x_{0}\right)}{\rho}+k_{\rho}
$$

Amplitude function is

$$
F\left(k_{\rho}\right)=k_{\rho}^{3 / 2} \cdot \frac{\varepsilon_{2} \kappa_{1}-\varepsilon_{1} \kappa_{2}}{\varepsilon_{2} \kappa_{1}+\varepsilon_{1} \kappa_{2}} .
$$

Next, according to the SPM method [11-13, 17], the "stationary point" is calculated from the following relation:

$$
f^{\prime}\left(k_{\rho}\right)=\frac{d f\left(k_{\rho}\right)}{d k_{\rho}}=0
$$




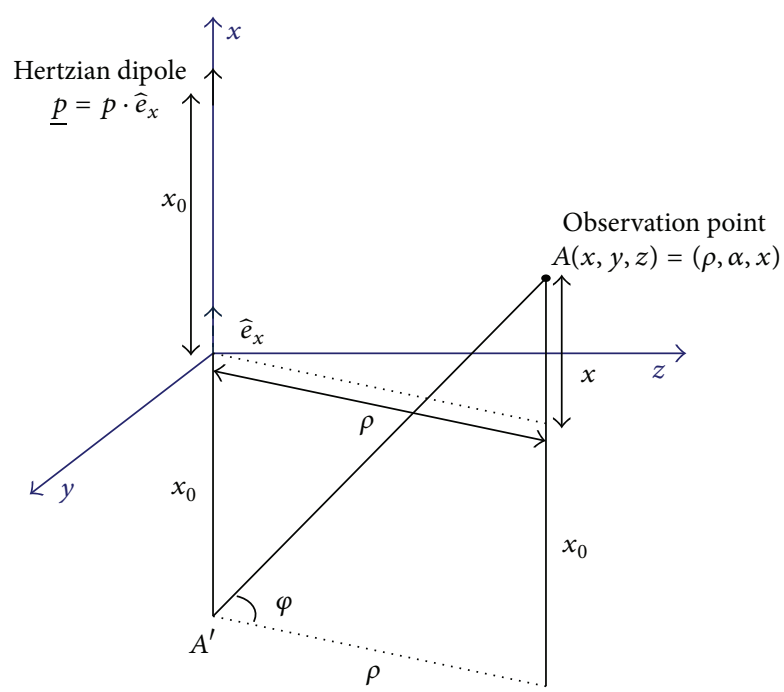

FIgURE 2: Geometry of the radiation problem considered in this paper, where also the image $A^{\prime}$ of the radiating Hertzian dipole is shown. Regarding angle $\varphi$ shown in this figure, $\cos \varphi$ is given by (41).

which finally yields the following expression for the "stationary point" (only one stationary point exists):

$$
\begin{aligned}
k_{\rho s} & =\frac{k_{01} \rho}{\left[\left(x+x_{0}\right)^{2}+\rho^{2}\right]^{1 / 2}} \\
& =k_{01} \frac{1}{\left[1+\left(\left(x+x_{0}\right) / \rho\right)^{2}\right]^{1 / 2}} \\
& =k_{01} \cos \varphi,
\end{aligned}
$$

where $\varphi$ is the angle defined by the image point of the radiating dipole, the observation point, and the horizontal line drawn from the above-mentioned image point and $\cos \varphi$ is given by (41). Furthermore, note that angle $\varphi$ is the wellknown "grazing angle" in the literature [15], as shown in Figure 2.

Note here that for the air-lossy ground problem considered here $k_{\rho s}$ is real and positive and $k_{\rho s}<k_{01}$. Also, we can easily see that

$$
\lim _{\rho \rightarrow \infty} k_{\rho s}=\lim _{\left(x+x_{0}\right) \rightarrow 0} k_{\rho s}=k_{01} .
$$

Furthermore, according to the SPM method [11-13, 17], we also have to calculate the second derivative of the phase function, which in our case is calculated, from (38) and (40) as

$$
f^{\prime \prime}\left(k_{\rho s}\right)=-\frac{\left(x+x_{0}\right)}{\rho} \cdot \frac{k_{01}^{2}}{\left(k_{01}^{2}-k_{\rho s}^{2}\right)^{3 / 2}} .
$$

Note here that $f^{\prime \prime}\left(k_{\rho}\right)$ is always negative; that is,

$$
\operatorname{sgn}\left[f^{\prime \prime}\left(k_{\rho s}\right)\right]=-1
$$

whose relation is needed in the application of SPM method.

Then, by actually applying the SPM method [11-13, 17], from (37), we find

$$
\begin{aligned}
I_{1}= & i F\left(k_{\rho s}\right) e^{i \rho f\left(k_{\rho s}\right)} \cdot e^{i(\pi / 4) \operatorname{sgn}\left[f^{\prime \prime}\left(k_{\rho s}\right)\right]} \sqrt{\frac{2 \pi}{\rho\left|f^{\prime \prime}\left(k_{\rho s}\right)\right|}} \\
& \cdot \sqrt{\frac{2}{\pi \rho}} \exp \left(\frac{i \pi}{4}\right)
\end{aligned}
$$

or

$$
I_{1}=\frac{i 2}{\rho} \frac{1}{\left|f^{\prime \prime}\left(k_{\rho s}\right)\right|^{1 / 2}} F\left(k_{\rho s}\right) e^{i \rho f\left(k_{\rho s}\right)}
$$

Then, by using expressions (33)-(34) and (45), we finally end up with the following expressions:

$$
\begin{aligned}
& I_{1}=\frac{i 2}{k_{01} \rho^{1 / 2}} \frac{1}{\left(x+x_{0}\right)^{1 / 2}} \kappa_{1 s}^{3 / 2} k_{\rho s}^{3 / 2} \frac{\varepsilon_{2} \kappa_{1 s}-\varepsilon_{1} \kappa_{2 s}}{\varepsilon_{2} \kappa_{1 s}+\varepsilon_{1} \kappa_{2 s}} e^{i k_{\rho s} \rho} e^{i \kappa_{1 s}\left(x+x_{0}\right)}, \\
& I_{2}=\frac{i 2}{k_{01} \rho^{1 / 2}} \frac{1}{\left(x+x_{0}\right)^{1 / 2}} \kappa_{1 s}^{1 / 2} k_{\rho s}^{5 / 2} \frac{\varepsilon_{2} \kappa_{1 s}-\varepsilon_{1} \kappa_{2 s}}{\varepsilon_{2} \kappa_{1 s}+\varepsilon_{1} \kappa_{2 s}} e^{i k_{\rho s} \rho} e^{i \kappa_{1 s}\left(x+x_{0}\right)}, \\
& I_{3}=\frac{i 2}{k_{01} \rho^{1 / 2}} \frac{1}{\left(x+x_{0}\right)^{1 / 2}} \kappa_{1 s}^{1 / 2} k_{\rho s}^{3 / 2} \frac{\varepsilon_{2} \kappa_{1 s}-\varepsilon_{1} \kappa_{2 s}}{\varepsilon_{2} \kappa_{1 s}+\varepsilon_{1} \kappa_{2 s}} e^{i k_{\rho s} \rho} e^{i \kappa_{1 s}\left(x+x_{0}\right)},
\end{aligned}
$$

where

$$
\kappa_{1 s}=\sqrt{k_{01}^{2}-k_{\rho s}^{2}}=k_{01} \sin \varphi,
$$

where angle $\varphi$ is defined in Figure 2, and

$$
\kappa_{2 s}=\sqrt{k_{02}^{2}-k_{\rho s}^{2}} .
$$

Then, our final solution in the high frequency regime (i.e., by using the SPM method) consists of (30)-(31) and (47)-(51), where $k_{\rho s}$ is given by (41). 


\section{Final Formulae for the Received Electric and Magnetic Field Vector: Fields Reflected from the Lossy Ground}

5.1. Electric Field Vector. By using (30), (47), and (48), we obtain the following result for the electric field vector, scattered from the lossy ground, at the observation point:

$$
\begin{aligned}
\underline{E}_{x>0}^{s c}= & \frac{p}{4 \pi \varepsilon_{0} \varepsilon_{r 1}} \frac{1}{\rho^{1 / 2}} \frac{1}{\left(x+x_{0}\right)^{1 / 2}} \frac{\kappa_{1 s}^{1 / 2} k_{\rho s}^{3 / 2}}{k_{01}} \\
& \cdot \frac{\varepsilon_{2} \kappa_{1 s}-\varepsilon_{1} \kappa_{2 s}}{\varepsilon_{2} \kappa_{1 s}+\varepsilon_{1} \kappa_{2 s}} e^{i k_{\rho s} \rho} e^{i \kappa_{1 s}\left(x+x_{0}\right)} \cdot\left(\kappa_{1 s} \widehat{e}_{\rho}+k_{\rho s} \widehat{e}_{x}\right) \\
= & \frac{p k_{01}}{4 \pi \varepsilon_{0} \varepsilon_{r 1}} \frac{(\sin \varphi)^{1 / 2}(\cos \varphi)^{3 / 2}}{\rho^{1 / 2}\left(x+x_{0}\right)^{1 / 2}} \\
& \cdot \frac{\varepsilon_{2} \kappa_{1 s}-\varepsilon_{1} \kappa_{2 s}}{\varepsilon_{2} \kappa_{1 s}+\varepsilon_{1} \kappa_{2 s}} e^{i k_{\rho s} \rho} e^{i \kappa_{1 s}\left(x+x_{0}\right)} \\
& \cdot\left(\kappa_{1 s} \widehat{e}_{\rho}+k_{\rho s} \widehat{e}_{x}\right) \\
= & \frac{p k_{01} \cos \varphi}{4 \pi \varepsilon_{0} \varepsilon_{r 1}\left(A^{\prime} A^{\prime}\right)} \cdot \frac{\varepsilon_{2} \kappa_{1 s}-\varepsilon_{1} \kappa_{2 s}}{\varepsilon_{2} \kappa_{1 s}+\varepsilon_{1} \kappa_{2 s}} e^{i k_{\rho s} \rho} e^{i \kappa_{1 s}\left(x+x_{0}\right)} \\
& \cdot\left(\kappa_{1 s} \widehat{e}_{\rho}+k_{\rho s} \widehat{e}_{x}\right)
\end{aligned}
$$

where angle $\varphi$ and distance $\left(A^{\prime} A^{\prime}\right)$ are shown in Figure 2 (note that $\left(A^{\prime} A^{\prime}\right)$ is the distance between the image point and the observation point and $\varphi$ is the so-called "grazing angle" [15]). Moreover, we observe that function

$$
R_{V}=\left|R_{V}\right| e^{i \varphi_{V}}=\frac{\varepsilon_{2} \kappa_{1 s}-\varepsilon_{1} \kappa_{2 s}}{\varepsilon_{2} \kappa_{1 s}+\varepsilon_{1} \kappa_{2 s}}
$$

is the usual (complex) "Fresnel reflection coefficient" for the "Sommerfeld radiation problem" considered in this paper (since $R_{V}$ is complex, this means change in magnitude and in phase of the incident EM wave upon reflection from the lossy ground).

Furthermore, in order to elaborate a little more in formula (52), we define the "amplitude factor" $F_{0}$ by

$$
F_{0}=\frac{p}{4 \pi} \frac{1}{\rho^{1 / 2}} \frac{1}{\left(x+x_{0}\right)^{1 / 2}} \frac{\kappa_{1 s}^{1 / 2} k_{\rho s}^{3 / 2}}{k_{01}}
$$

and the "phase factor" $\varphi_{0}$ by

$$
\varphi_{0}=k_{\rho s} \rho+\kappa_{1 s}\left(x+x_{0}\right)
$$

which is the phase in (52) in addition to the phase $\varphi_{V}$ originating from the complex "Fresnel reflection coefficient" $R_{V}$ of (53). Then, from (52)-(55), we obtain

$$
\underline{E}_{x>0}=\frac{1}{\varepsilon_{0} \varepsilon_{r 1}} F_{0} R_{V} e^{i \varphi_{0}}\left(\kappa_{1 s} \widehat{e}_{\rho}+k_{\rho s} \widehat{e}_{x}\right) .
$$

Finally, by taking (41) and (50) into account, we find the following expressions for horizontal (along $\widehat{e}_{\rho}$ ) and vertical (along $\widehat{e}_{x}$ ) components of electric field vector, respectively:

$$
\begin{gathered}
\left.E_{h}^{\mathrm{sc}}\right|_{x>0}=\frac{\kappa_{1 s}}{\varepsilon_{0} \varepsilon_{r 1}} F_{0} R_{V} e^{i \varphi_{0}}=\frac{k_{01} \sin \varphi}{\varepsilon_{0} \varepsilon_{r 1}} F_{0} R_{V} e^{i \varphi_{0}}, \\
\left.E_{V}^{\mathrm{sc}}\right|_{x>0}=\frac{k_{\rho s}}{\varepsilon_{0} \varepsilon_{r 1}} F_{0} R_{V} e^{i \varphi_{0}}=\frac{k_{01} \cos \varphi}{\varepsilon_{0} \varepsilon_{r 1}} F_{0} R_{V} e^{i \varphi_{0}}, \\
\left|\underline{E}_{\mathrm{tot}}^{\mathrm{sc}}\right|_{x>0}=\sqrt{\left|E_{h}^{\mathrm{sc}}\right|^{2}+\left|E_{V}^{\mathrm{sc}}\right|^{2}}=\frac{k_{01}}{\varepsilon_{0} \varepsilon_{r 1}} F_{0}\left|R_{V}\right| .
\end{gathered}
$$

5.2. Magnetic Field Vector. Similarly, by using (31) and (49), we find the following expression for the scattered magnetic field vector above the flat and lossy ground:

$$
\begin{aligned}
\underline{H}_{x>0}^{s c}= & \frac{\omega p}{4 \pi} \frac{1}{\rho^{1 / 2}} \frac{1}{\left(x+x_{0}\right)^{1 / 2}} \frac{\kappa_{1 s}^{1 / 2} k_{\rho s}^{3 / 2}}{k_{01}} \\
& \cdot \frac{\varepsilon_{2} \kappa_{1 s}-\varepsilon_{1} \kappa_{2 s}}{\varepsilon_{2} \kappa_{1 s}+\varepsilon_{1} \kappa_{2 s}} e^{i k_{\rho s} \rho} e^{i \kappa_{1 s}\left(x+x_{0}\right)} \widehat{e}_{\alpha} \\
= & \frac{\omega k_{01} p}{4 \pi} \frac{(\sin \varphi)^{1 / 2}(\cos \varphi)^{3 / 2}}{\rho^{1 / 2}\left(x+x_{0}\right)^{1 / 2}} \frac{\varepsilon_{2} \kappa_{1 s}-\varepsilon_{1} \kappa_{2 s}}{\varepsilon_{2} \kappa_{1 s}+\varepsilon_{1} \kappa_{2 s}} \\
& \times e^{i k_{\rho s} \rho} e^{i \kappa_{1 s}\left(x+x_{0}\right)} \widehat{e}_{\alpha} \\
= & \frac{\omega k_{01} p \cdot \cos \varphi}{4 \pi\left(A^{\prime} A^{\prime}\right)} \frac{\varepsilon_{2} \kappa_{1 s}-\varepsilon_{1} \kappa_{2 s}}{\varepsilon_{2} \kappa_{1 s}+\varepsilon_{1} \kappa_{2 s}} e^{i k_{\rho s} \rho} e^{i \kappa_{1 s}\left(x+x_{0}\right)} \widehat{e}_{\alpha} .
\end{aligned}
$$

Furthermore, by using the definitions of quantities $R_{V}, F_{0}$, and $\varphi_{0},(53)-(55)$, we obtain

$$
\begin{gathered}
\left.\underline{H}^{\mathrm{sc}}\right|_{x>0}=\omega F_{0} R_{V} e^{i \varphi_{0}} \widehat{e}_{\alpha}, \\
\left|\underline{H}^{\mathrm{sc}}\right|_{x>0}=\omega F_{0}\left|R_{V}\right| .
\end{gathered}
$$

Finally, note that from (58) and (61) it follows that

$$
\frac{\left|\underline{E}_{\mathrm{tot}}^{\mathrm{sc}}\right|_{x>0}}{\left|\underline{H}^{\mathrm{sc}}\right|_{x>0}}=\zeta=\sqrt{\frac{\mu_{0}}{\varepsilon_{0}}},
$$

where $\zeta$ is the free space impedance.

Expressions (52) and (59) are the classical expressions for the EM field reflected from the lossy ground and originating from the image point, as shown in Figure 2. Then, by using the newly derived expressions for the received EM field in spectral domain in this paper, (28), and by applying the SPM method, that is, in the high frequency regime, the classical "space wave" in region $x>0$ [15] is derived. This result has the following two interesting consequences.

(1) The "space wave" [15], which corresponds to the complex summation (interference) of the reflected fields, (52) and (59), and the line-of-sight (LOS) fields (the latter not included in these equations, but shown in (15) and (18)), is the solution to the Sommerfeld radiation problem in the high frequency regime, where the so-called "surface wave" can be ignored $[7,8,15]$. 


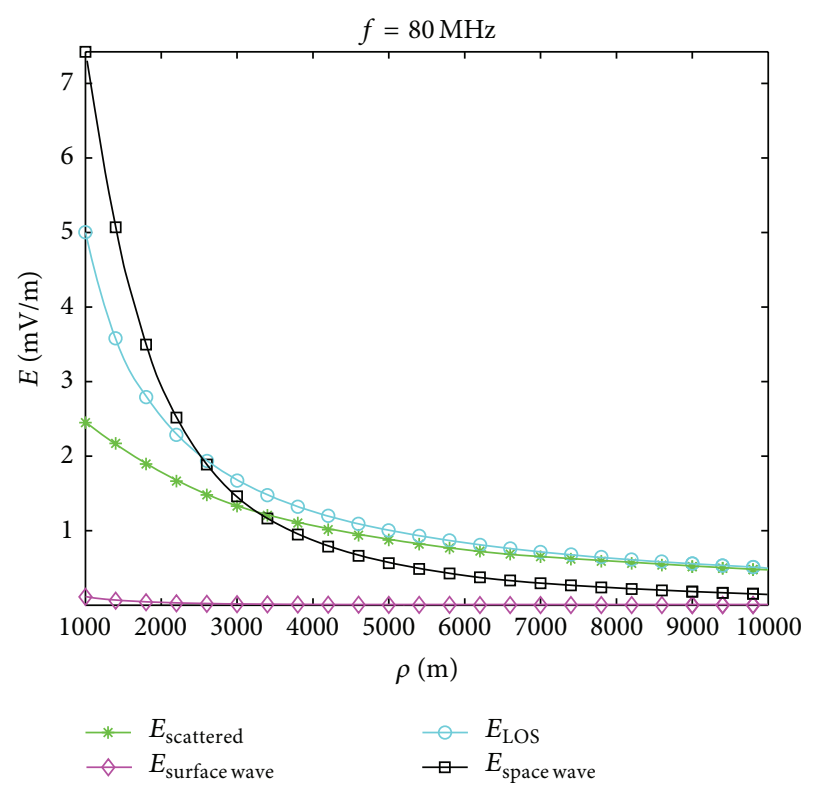

FIGURE 3: Electric fields at observation point as a function of horizontal distance $\rho$ between transmitting Hertzian dipole and observation point, for frequency $f=80 \mathrm{MHz}$. Here, the various components of received electric field are shown as follows: lineof-sight (LOS) field (circle), field scattered from ground (asterisk), "space wave" (square), and "surface wave" (diamond). Note that in this case Norton's "surface wave" is rather negligible as compared to the corresponding "space wave" [15].

(2) The validity of expressions (28) for the scattered EM field above the lossy and flat ground, derived in a novel way in this paper, has been confirmed in all aspects in the high frequency regime. Then, it appears that expressions (28)-(29) represent a very convenient starting point for further research with respect to the calculation of the received EM field for any frequency of the radiating dipole (i.e., including also low frequency effects), either above or below the ground, in an exact analytical way using the "residue theorem" [16], or in a numerical way (i.e., through numerical integration techniques).

\section{Numerical Results in the High Frequency Regime: Comparison with Norton's Results}

In this Section, indicative numerical results are provided for the electric field (magnitude) at the receiver point as a function of the horizontal distance $(\rho)$ between transmitting Hertzian dipole and receiver position. These numerical results include the electric field scattered from the ground, magnitude of (52), the line-of-sight (LOS) field, the socalled "space wave" (which is just the complex summation (interference) of the two fields mentioned above), and, finally, the so-called "surface wave," according to Norton [7, 8, 15]. Furthermore, these numerical results are provided for frequency of radiating dipole $f=80 \mathrm{MHz}$ (Figure 3) or $f=30 \mathrm{MHz}$ (Figure 4).

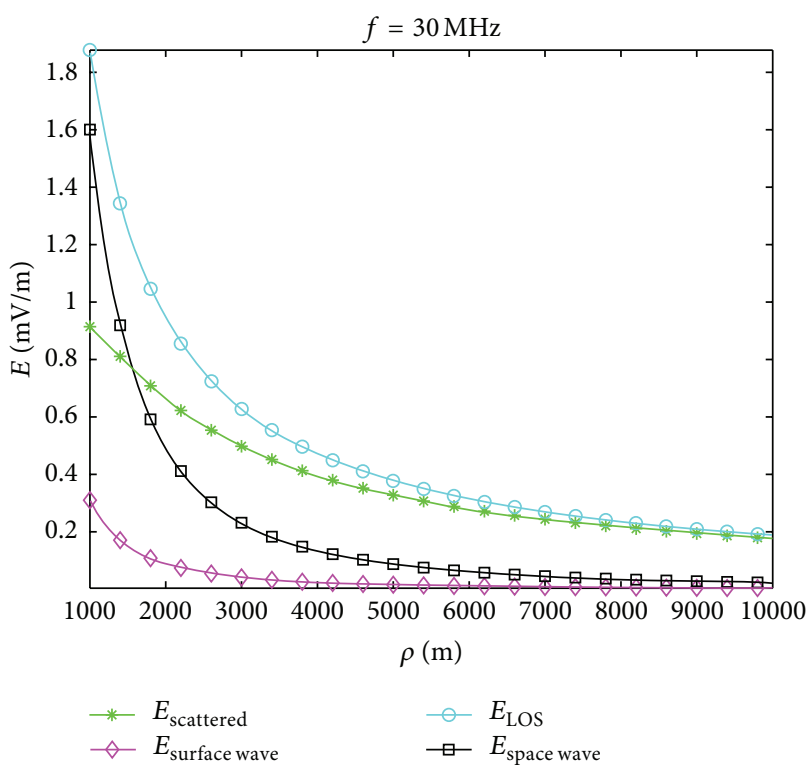

FIgURE 4: Similar to Figure 3, except that here the frequency of radiating Hertzian dipole is now equal to $30 \mathrm{MHz}$ (lower frequency). In this case of lower frequency, the "surface wave" cannot be considered negligible, as compared to the "space wave" [15].

Comparison of numerical results for LOS electric field, scattered electric field, and space wave, derived from our formulation, and Norton's results $[7,8,15]$ shows very good agreement, as it can be seen in Figures 3 and 4. The surface wave represented in Figures 3 and 4 is the so-called "Norton surface wave" $[7,8,15]$. Note that at the higher frequency of $80 \mathrm{MHz}$ (Figure 3) the surface wave, according to Norton's formulation $[7,8,15]$, is rather negligible, as compared to the "space wave," while it becomes rather more important at the lower frequency of $30 \mathrm{MHz}$ (Figure 4). Our proposed SPM method of Sections 4 and 5 (which is inherently a "high frequency method") ignores this surface wave contribution in the high frequency regime.

Moreover, note that the problem parameters in Figures 3 and 4 are selected as follows: height of transmitting dipole $x_{0}=60 \mathrm{~m}$, height of observation point (receiver position) $x=$ $15 \mathrm{~m}$, current of the radiating Hertzian dipole $I=1 \mathrm{~A}$, length of the Hertzian dipole $2 h=0.1 \mathrm{~m}$ (much smaller than the wavelength $\lambda=c / f$ in both cases), relative dielectric constant of ground $\varepsilon_{r}=20$, and ground conductivity $\sigma=0.01 \mathrm{~S} / \mathrm{m}$. Finally, note that the relation between current $I$ and dipole moment $p$ is given by $I(2 h)=i \omega p$, where $\omega=2 \pi f$ and $i$ is the unit imaginary number.

\section{Conclusions: Future Research}

In this paper, we formulated the radiation problem from a vertical short (Hertzian) dipole above flat and lossy ground in the spectral domain, which resulted in an easy-to-manipulate integral expression for the received EM field above or below the ground. As also explained above, this formulation appears to have inherent advantages over the classical formulation by Sommerfeld [6], since it avoids the use of the so-called Hertz 
potential and its subsequent differentiation for the calculation of the received EM field. Subsequently, by applying the stationary phase method (SPM) in the high frequency regime, the classical solution for the "space wave" was rederived in a new fashion, thus showing that this is the dominant solution in this high frequency regime. Mathematical derivations regarding the application of our proposed method in spectral domain, as well as the application of the SPM method, were provided in reasonable detail above. Finally, numerical results in this high frequency limit were obtained and they were compared to Norton's results $[7,8,15]$.

Corresponding research in the near future by our research group will concentrate on the calculation of the received EM field below the ground at the high frequency regime (by using again the SPM method). Furthermore, we will calculate the received EM field, above or below the ground, for any frequency of the radiating dipole, in an exact and analytical manner [16] or in a numerical way (i.e., through the use of numerical integration techniques [18]). In this context, the behavior of surface waves will become evident through the use of the residue theorem, when applied to (28), in a way similar to [6].

Moreover, we intend also to investigate the formulation of the same radiation problem in spectral domain, but now in the case of a horizontal radiating Hertzian dipole above flat and lossy ground. In addition, further investigations will be performed in the case of rough (and not flat) ground and in the case of curvature of the earth's surface for large distance communication applications. Finally, in the near future, our research group will focus on the design of a software product for accurate prediction of pass loss in different types of environment, like urban, suburban, and rural environments. The above software tool will be based on the exact electromagnetic (EM) method proposed in this paper, and therefore it is expected that it will exhibit important advantages over previously developed corresponding software tools. In this framework, comparisons with existing commercial software tools will also be performed [19].

\section{Conflict of Interests}

The authors declare that there is no conflict of interests regarding the publication of this paper.

\section{References}

[1] A. N. Sommerfeld, "Propagation of waves in wireless telegraphy," Annals of Physics, vol. 28, pp. 665-736, 1909.

[2] A. N. Sommerfeld, "Propagation of waves in wireless telegraphy," Annals of Physics, vol. 81, pp. 1135-1153, 1926.

[3] J. R. Wait, "The ancient and modern history of EM groundwave propagation," IEEE Antennas and Propagation Magazine, vol. 40, no. 5, pp. 7-24, 1998.

[4] R. J. King, "EM wave propagation over a constant impedance plane," Radio Science, vol. 4, pp. 225-268, 1969.

[5] J. Zenneck, "Propagation of plane EM waves along a plane conducting surface," Annals of Physics, vol. 23, pp. 846-866, 1907.
[6] T. K. Sarkar, W. Dyab, M. N. Abdallah et al., "Electromagnetic macro modeling of propagation in mobile wireless communication: theory and experiment," IEEE Antennas and Propagation Magazine, vol. 54, no. 6, pp. 17-43, 2012.

[7] K. A. Norton, "The propagation of radio waves over the surface of the earth," Proceedings of the IRE, vol. 24, pp. 1367-1387, 1936.

[8] K. A. Norton, "The propagation of radio waves over the surface of the earth and in the upper atmosphere," Proceedings of the Institute of Radio Engineers, vol. 25, no. 9, pp. 1203-1236, 1937.

[9] S. Sautbekov, "The generalized solutions of a system of Maxwell's equations for the uniaxial anisotropic media," in Electromagnetic Waves Propagation in Complex Matter, A. A. Kishk, Ed., chapter 1, pp. 3-24, InTech, Zagreb, Croatia, 2011.

[10] S. Sautbekov, R. Kasimkhanova, and P. Frangos, "Modified solution of Sommerfeld's problem," in Proceedings of the International Conference on Communications, Electromagnetics and Medical Applications (CEMA '10), pp. 5-8, National Technical University of Athens (NTUA), Athens, Greece, 2010.

[11] C. A. Balanis, "Appendix VIII: method of stationary phase," in Antenna Theory: Analysis and Design, pp. 922-927, John Wiley \& Sons, New York, NY, USA, 1997.

[12] H. Moschovitis, Asymptotic methods and high frequency techniques for the calculation of electromagnetic scattering by using the modified stationary phase method [Doctoral dissertation], National Technical University of Athens (NTUA), Athens, Greece, 2010, (Greek).

[13] C. G. Moschovitis, K. T. Karakatselos, E. G. Papkelis et al., "Scattering of electromagnetic waves from a rectangular plate using an enhanced stationary phase method approximation," IEEE Transactions on Antennas and Propagation, vol. 58, no. 1, pp. 233-238, 2010.

[14] http://arxiv.org/abs/1401.1720.

[15] J. Fikioris, Introduction to Antenna Theory and Propagation of Electromagnetic Waves, National Technical University of Athens (NTUA), Athens, Greece, 1982 (Greek).

[16] G. Arfken, Mathematical Methods for Physists, Academic Press, Orlando, Fla, USA, 3rd edition, 1985.

[17] C. G. Moschovitis, H. T. Anastassiu, and P. V. Frangos, "Scattering of electromagnetic waves from a rectangular plate using an extended stationary phase method based on Fresnel functions (SPM-F)," Progress in Electromagnetics Research, vol. 107, pp. 6399, 2010.

[18] M. Abramowitz and I. A. Stegun, Handbook of Mathematical Functions, Dover, New York, NY, USA, 10th edition, 1972.

[19] A. Hrovat, "An open-source radio coverage prediction tool," in Proceedings of the 14th WSEAS International Conference on Communications (ICCOM '10), pp. 135-140, 2010. 

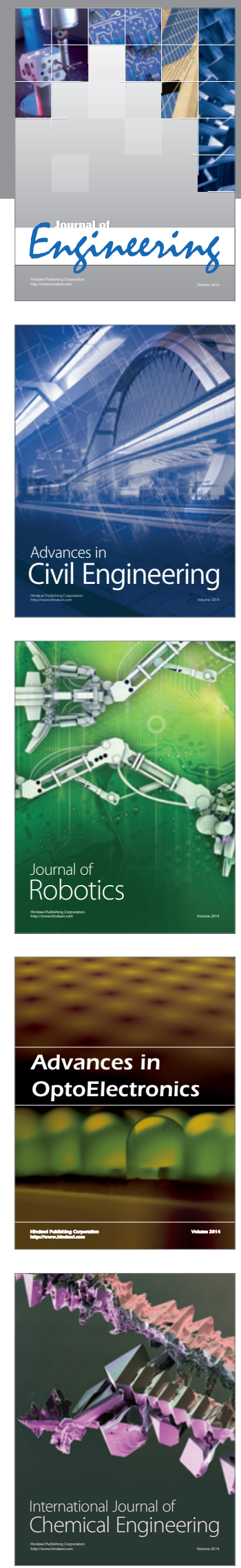

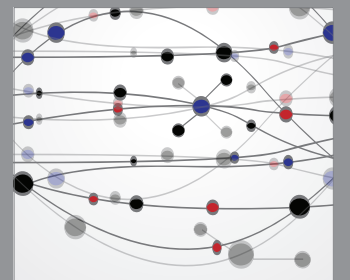

The Scientific World Journal
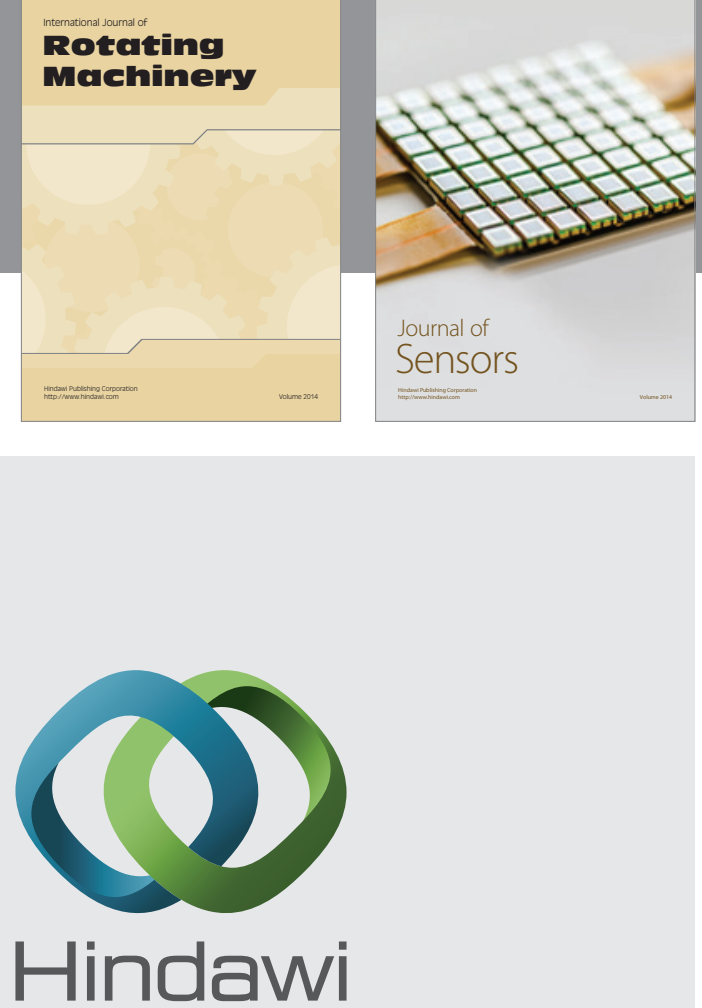

Submit your manuscripts at http://www.hindawi.com
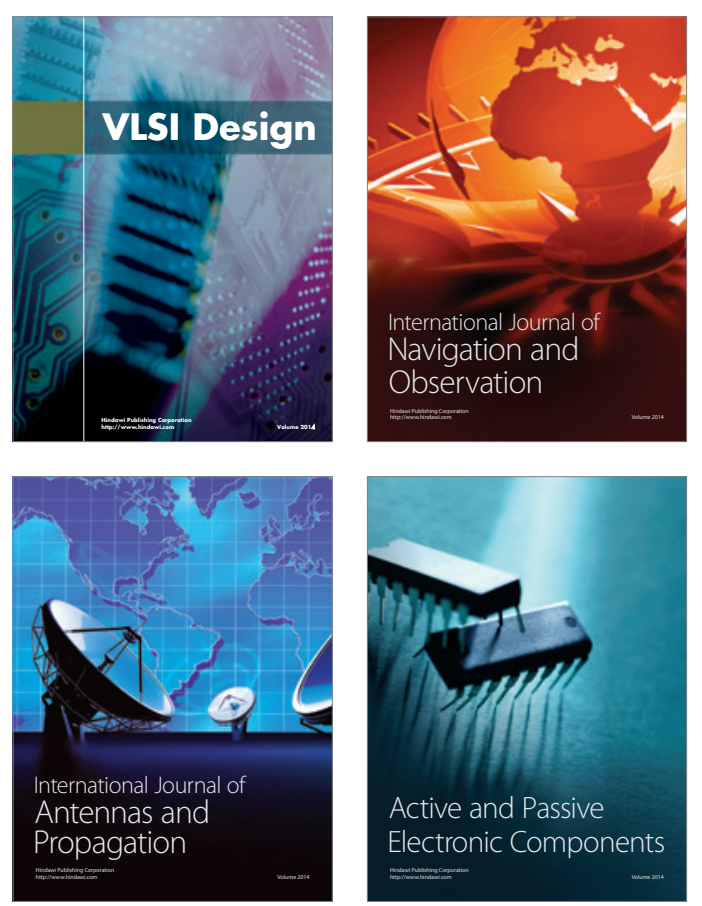
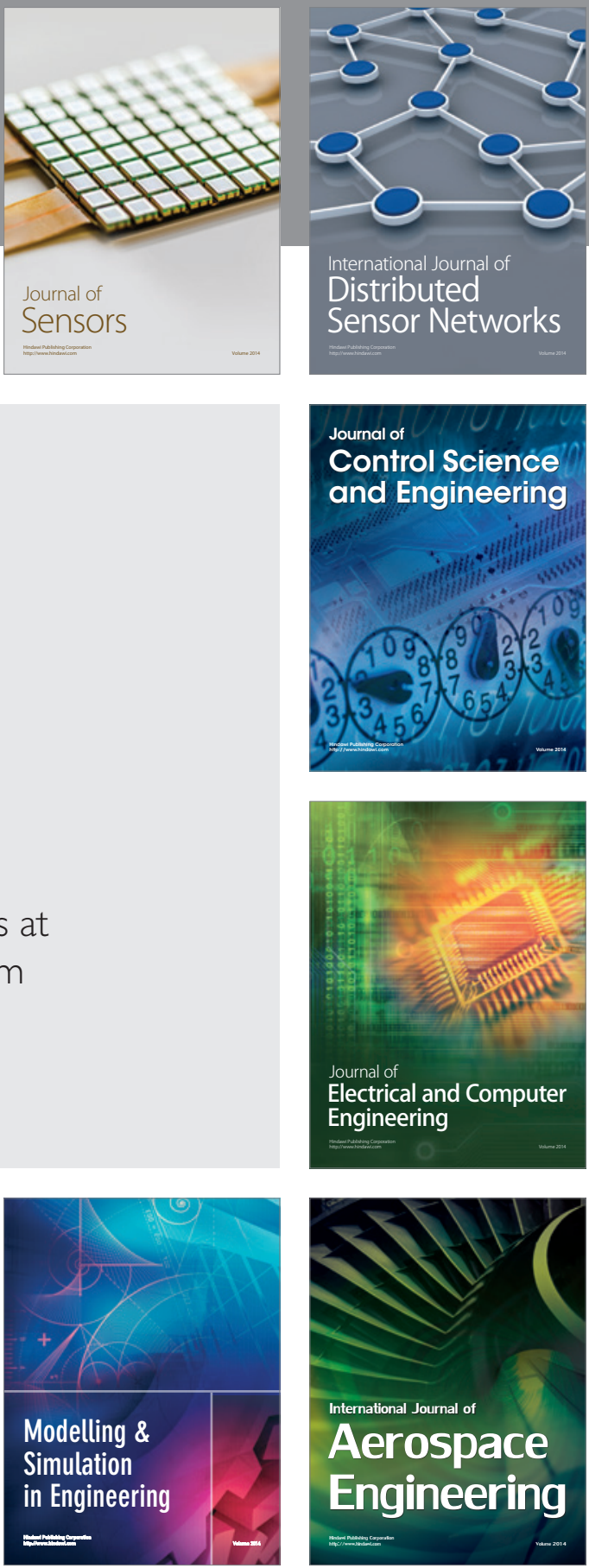

Journal of

Control Science

and Engineering
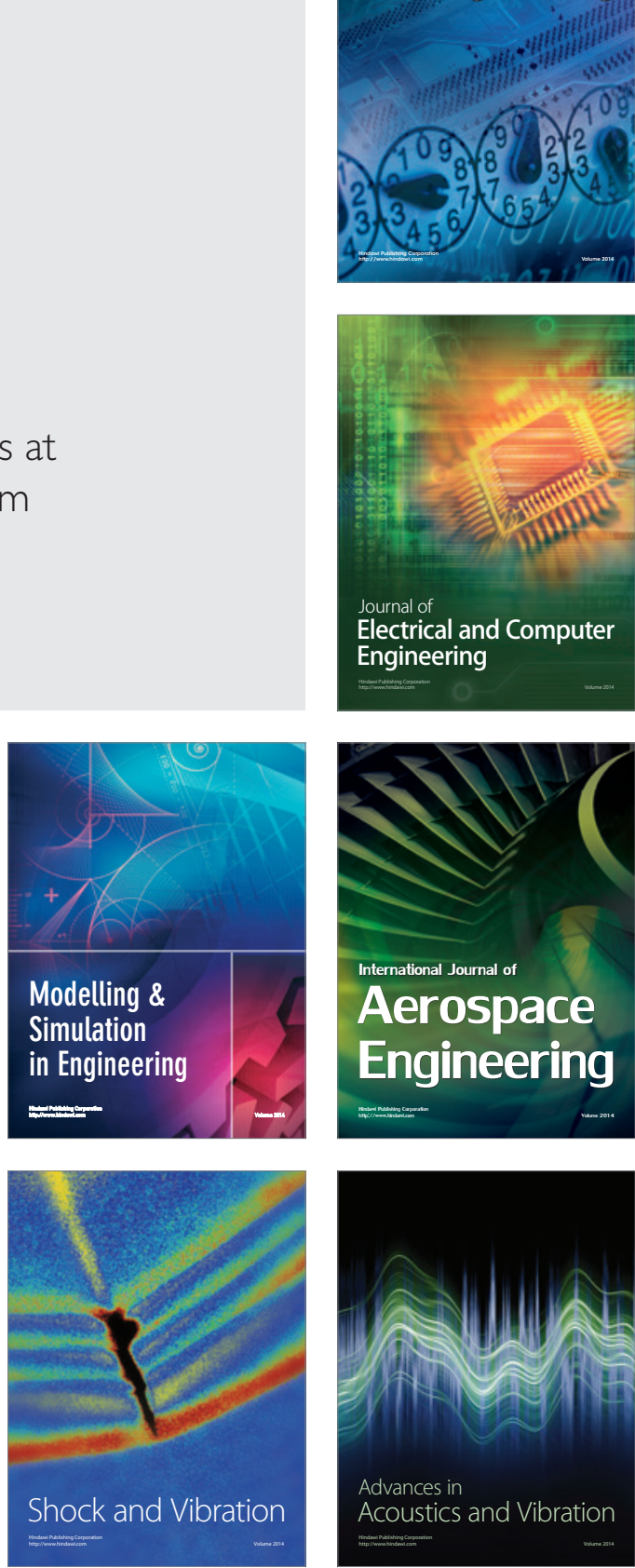